\title{
SCIDOC
}

\author{
International Journal of Dentistry and Oral Science (IJDOS) \\ ISSN: 2377-8075
}

\section{The Effects of Xylitol Gum on the Bacterial Composition of Saliva and Plaque}

\section{Research Article}

Rafeek $\mathrm{R}^{1 *}$, Akpaka PE ${ }^{2}$, Bishop $\mathrm{AK}^{1}$, Peters $\mathrm{T}^{1}$, Bandoo $\mathrm{C}^{1}$, Seunath $\mathrm{S}^{1}$, Addae $\mathrm{J}^{3}$

${ }^{1}$ School of Dentistry, Faculty of Medical Sciences, The University of the West Indies, St. Augustine, Trinidad and Tobago.

${ }^{2}$ Department of Paraclinical Sciences, Faculty of Medical Sciences, The University of the West Indies, St. Augustine, Trinidad and Tobago.

${ }_{3}^{3}$ Department of Preclinical Sciences, Faculty of Medical Sciences, The University of the West Indies, St. Augustine, Trinidad and Tobago.

\section{Abstract}

\begin{abstract}
Aim: The objective of this study was to investigate the short-term consumption effect of xylitol gum on the bacterial composition of saliva and plaque flora in a cohort of healthy adult individuals.

Methods: A randomized controlled double blind study was carried out on 29 healthy volunteers recruited from the university student population after screening them for Mutans Streptococci group. These fulfilled the study criteria and successfully enrolled in the study. Experimental gum (Epic spearmint) and Control gum (Eclipse spearmint) were given to these cohort of students alternatively following which their saliva and plaque specimens $(n=116$, saliva $n=58$, plaque $n=58)$ were assayed for their bacterial flora using standard microbiological procedures.

Results: A hundred and ten bacterial organisms were recovered from plaque and saliva of these participants. The Mutans Streptococci group (Streptococcus mutans and Streptococcus sobrinus) were the most $(68.2 \%)$ prevalent bacteria recovered. While Streptococcus mutans were mostly recovered in both groups, (12 vs 9), there was no statistically significant difference in the colony count of bacteria in each person before and after chewing sorbitol gum ( $\mathrm{p}=0.125$; Wilcoxon Signed Rank test). The trend was different with those that chewed xylitol gum as bacteria colony count in the saliva and plaque of each person before and after using xylitol gum showed significant decreases in the counts $(\mathrm{p}<0.001)$ in both cases (Wilcoxon Signed Rank test).

Conclusion: The study confirms the fact xylitol reduces the oral bacterial flora which supports the argument that this helps reduce the prevalence of dental caries.
\end{abstract}

Keywords: Xylitol Gum; Sorbitol; Oral Bacteria.

\section{Introduction}

The modern concept of caries is an interaction between genetic and environmental factors where biological, social, behavioral and psychological factors play essential roles together with that of the dental biofilm [1]. There is an association between dental caries (decay) and the presence of Streptococcus mutans and Streptococcus sobrinus and also lactobacilli in saliva and plaque [2]. Streptococcus mutans and Streptococcus sobrinus species are often referred to collectively as the Mutans streptococci (MS) [3]. In contrast, Streptococcus sanguinis and Streptococcus mitis are oral bacteria associated with tooth surfaces free of dental caries and are thought to be beneficial to oral health [4]. There is still a lot to be learnt about the roles of the oral bacterial flora with more than 700 bacterial species identified and $35 \%$ of them not yet cultured and characterized [5].

The prevention of dental caries has focused on the use of sugar substitutes including polyols or non-fermentable sweeteners of which the most common are sorbitol and xylitol [6]. Xylitol is a naturally occurring carbohydrate sweetener found in fruits and vegetables. Xylitol is a nutritive sweetener, non-fermentable by oral bacteria and has been shown to have microbiological properties. Xylitol disrupts the energy production leading to bacterial cell death, reduces the acid producing potential of bacteria and their adhesion to the tooth surface [7]. Sorbitol is a six-carbon polyol and is thought to be fermented to a small degree unlike xylitol [8]. The use of xylitol containing chewing gum has been reported to

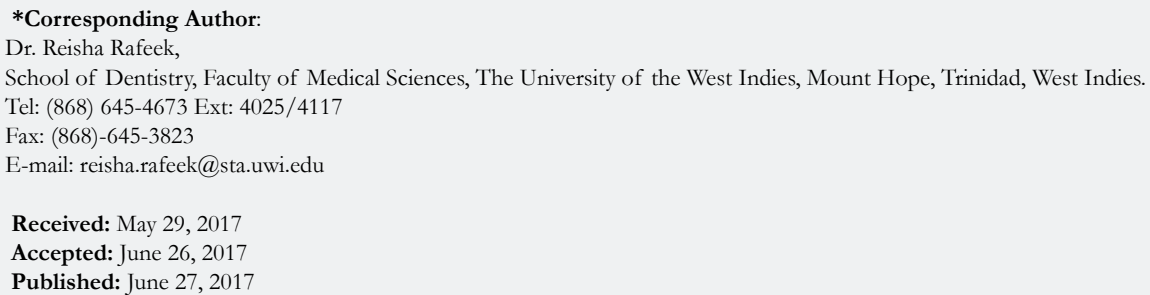

Copyright: Rafeek $\mathbf{R}^{\circ}$ 2017. This is an open-access article distributed under the terms of the Creative Commons Attribution License, which permits unrestricted use, distribution and reproduction in any medium, provided the original author and source are credited. 
reduce the level of Mutans streptococci (MS) and lactobacilli in saliva and plaque [9-11]. However, some studies have found no effects of xylitol consumption on either salivary MS or lactobacilli [12, 13]. There is hardly any information on the effects of xylitol on the caries-protective bacteria of Streptococcus sanguinis and Streptococcus mitis. A systematic review of clinical trials of xylitol versus sorbitol containing gum and syrup concluded that the evidence to support the greater effectiveness of xylitol over sorbitol was contradictory [14].

Hence, the aim of the current study was to investigate the effect of short-term consumption of xylitol gum on the bacterial composition of saliva and plaque flora in a cohort of healthy adult subjects.

\section{Materials And Methods}

\section{Subjects}

Healthy volunteers ( $n=30$, male 15 , females 15$)$ recruited from the university student population participated in this randomised, controlled, double-blind study. The subjects were examined and screened for salivary Streptococcus mutans, (Dentocult SM strip Mutans tests, Orion Diagnostic) to ensure the presence of MS.

\section{Inclusion Criteria}

Only subjects who were willing to comply with the study procedures were recruited. Also, subjects must have at least 20 teeth and gave written informed consent.

\section{Exclusion Criteria}

Subjects with the following were excluded from the study: systemic, infectious or inflammatory disease in the last month, taking medicines, antibiotics or fluoride in the last month, habitual consumers of xylitol or sorbitol-containing products and mouth rinses, abnormal salivary flow rates $(<1 \mathrm{ml} / \mathrm{min})$, pregnancy or the use of contraceptive pill, or particular dietary habits. One subject (male) started using antibiotics during the study and was then excluded.

\section{Ethical Approval}

The study was approved by the ethics committee of the University of the West Indies, Informed consent was obtained from participants at the initial visit before baseline saliva samples were taken.

\section{Experimental and Control Chewing Gums}

The experimental gum (1.5g/pellet) contained 70\% xylitol (Epic spearmint). The control gum (Eclipse spearmint) was the same as the xylitol gum but contained sorbitol as the main sweetener at $63 \%$ concentration and $2 \%$ maltilol. The gums were packed in colour-coded (red and blue) plastic containers. The colour codes were kept confidential during the data collection phase of the study.

\section{Study Design}

The study lasted 7 weeks altogether. During this period the sub- jects were instructed not to use antimicrobial medications, mouth washes or xylitol products, to consume their normal diet and continue their usual tooth brushing. Subjects were randomly allocated to one of two groups. Both groups entered a 4-week washout period followed by a 3-week treatment period. Group M used gum $A$ the red-coded gum for the treatment period and Group $\mathrm{F}$ used gum B. The subjects were instructed to use 2 gum pieces, three times daily after meals for both three-week treatment periods. The gum was not used during the washout period. Recommended chewing time was 6 minutes. They were instructed not to brush their teeth or use any other oral hygiene procedures at least 24 hours before sample collection and not to eat or drink at least 1 hour prior to collection.

\section{Sample Collection}

Saliva: Subjects chewed sterile paraffin wax in order to stimulate saliva production. The saliva was collected for 5 minutes in sterile tubes at the beginning and end of the treatment period to yield a total of 2 saliva samples per subject volunteer. The saliva was transferred into sterile flat-top Falcon labelled $50 \mathrm{ml}$ conical centrifuge tubes and sent to the microbiology laboratory of the Faculty of Medical Sciences on the same day for processing. In cases where this was not possible, samples were stored overnight in the refrigerator and sent to the laboratory the following day.

Plaque: Supra gingival plaque was collected from 6 teeth with a curette and the tip of the curette immersed in the labelled tube containing the sterile EDTA buffer for 4-5 seconds, wiping off the curette on the inside of the collection tube. The lid was replaced and the tube shaken for 4-5 seconds in an attempt to maximize dispersion of the specimen in the fluid. The tube was then placed in a ziploc bag and transported in ice immediately to the microbiology laboratory for analyses.

\section{Microbiological Analysis}

Both saliva and plaque specimens from the subjects were processed using standard microbiological procedures [15]. Briefly, the specimens were plated on blood, McConkey, Chocolate and Sabourauds agar and incubated in both aerobic and anerobic conditions at $35^{\circ} \mathrm{C}$ for $18-24$ hours. Observed positive growths were initially assessed microscopically by Gram stain, and preliminary biochemical tests (catalase, oxidase, coagulase etc.) were carried out on the recovered bacterial isolates. The automated system, Microscan WalkAway-96 (Beckman Coulter, Inc.) was used to identify the bacteria at species level. All procedures were performed according to the manufacturer's recommendations. The results were noted and analyzed.

\section{Results}

\section{Microbiology Results}

A total of 116 specimens ( saliva $=58$ and plaques $=58$ ) were collected from 29 subjects who fulfilled the study criteria and successfully participated in the study. Two sets of the specimens (saliva and plaques) were collected from each cohort. A total of 110 bacterial organisms were recovered from all the specimens analyzed (Table 1) and this revealed that Mutans Streptococci group (Streptococcus mutans and Streptococcus sobrinus) were the most preva- 
lent accounting for $68.2 \%$. These were distantly followed by Streptococcus pneumoniae 10\%. Enterobacter cloacae and Streptococcus pyogenes were the least with $2 \%$ each.

Microbiology analysis of saliva from the participants in the study before and after chewing sorbitol gum (Table 2) revealed that the bacterial agents encountered were almost the same species but differed in numbers. Mutans Streptococci group of bacteria were mostly recovered in both groups, $(12 \mathrm{v} 9)$, Klebsiella species $(3 \mathrm{v}$ 2), Streptococcus pneumoniae $(2 \mathrm{v} 2)$ and Pseudomonas species $(1 \mathrm{v}$ 1). There was no statistically significant difference in the colony count of bacteria in each person before and after chewing sorbitol gum ( $p=0.125$; Wilcoxon Signed Rank test).

A similar trend was observed for plaque specimens taken before and after the subjects chewed the sorbitol gum (Table 2). The distribution of organisms was Mutans Streptococci group of organisms (11 v 8), Klebsiella species (2 v 0), Pseudomonas species (1 v 0), Streptococcus pneumoniae (1 $\mathrm{v} 1)$ and Staphylococcus species (1 $\mathrm{v} 1)$. As in the analysis of the saliva, there was no statistically significant difference in the colony count of bacteria in the plaque following the chewing of sorbitol gum ( $\mathrm{p}=0.063)$.

The trends after subjects chewed xylitol gum were different from subjects after they chewed sorbitol. (Table 3). A statistical analysis of the bacteria colony count in the saliva and plaque of each person before and after using xylitol gum showed significant decreases in the counts $(\mathrm{p}<0.001)$ in both cases (Wilcoxon Signed Rank test).

\section{Discussion}

In this study we set out to explore potential use of xylitol gum as an anti-cariogenic agent. We investigated the distribution of salivary and plaque levels of harmful and protective bacterial organisms following the use of xylitol. Xylitol chewing gum has been reported to significantly reduce the oral normal flora especially the Mutans Streptococci levels in saliva and plaques $[9-11,14,16]$.

Our results show that most of the bacteria recovered from our participants were the Mutans streptococci $(68.2 \%)$. This result is not surprising but supports the knowledge that Streptococcus mutans is a major component of the oral and gut flora where they offer protection against periodontitis $[4,17]$.

An interesting significant finding from our analysis was that chewing xylitol gum did greatly suppress the number of S.mutans among our participants in the study. This is in agreement with findings reported by other researchers from several other places $[11,16,18,19]$ but is in contrast to other studies finding no change $[12,13]$.

Our study clearly shows that chewing sorbitol gum however did not decrease or reduce number of S.mutans in the oral cavity and this is in agreement with Bahador et al., 2012 [19] where sorbitol did not reduce S.mutans, S.sobrinus. S. sanguinis or S.mitis. This therefore may imply that chewing xylitol gum would be more beneficial to maintaining a healthy oral cavity flora than sorbitol gum in short term consumption.

Table 1. Distribution of Bacterial Organisms Recovered from the 116 Clinical Specimens (saliva and plaques).

\begin{tabular}{|c|c|c|}
\hline Organism & Number isolates & Percentage \\
\hline Mutans streptococci group & 75 & 68.2 \\
\hline Streptococcus pneumoniae & 11 & 10.0 \\
\hline Klebsiella species & 7 & 6.4 \\
\hline Staphylococcus species & 6 & 5.5 \\
\hline Pseudomonas species & 4 & 3.6 \\
\hline Serratia marscences & 3 & 2.7 \\
\hline Enterobacter cloacea & 2 & 1.8 \\
\hline Streptococcus pyogenes & 2 & 1.8 \\
\hline Total & 110 & 100 \\
\hline
\end{tabular}

Table 2. Distribution of Bacterial Organisms Recovered from saliva and plaque Specimens of Volunteers before and after Chewing Sorbitol Gum.

\begin{tabular}{|c|c|c|}
\hline Organisms & Recovered before & Recovered after \\
\hline & \multicolumn{2}{|c|}{ Saliva specimen 1 } \\
\hline Mutans Streptococci group & 12 & 9 \\
\hline Klebsiella spp & 3 & 2 \\
\hline Pseudomonas spp & 1 & 1 \\
\hline Strep pneumoniae & 2 & 2 \\
\hline & \multicolumn{2}{|c|}{ Plaque specimen 1 } \\
\hline Mutans Streptococci & 11 & 0 \\
\hline Klebsiellaspp & 2 & 0 \\
\hline Pseudomonas spp & 1 & 1 \\
\hline Streppneumoniae & 1 & 1 \\
\hline Staphylococcus spp & 1 & \\
\hline
\end{tabular}


Table 3. Distribution of Bacterial Organisms Recovered from saliva and plaque Specimens of Volunteers before and after Chewing Xylitol Gum.

\begin{tabular}{|c|c|c|}
\hline Organisms & Recovered before & Recovered after \\
\hline & \multicolumn{2}{|c|}{ Saliva specimen 2} \\
\hline Mutans Streptococci group & 11 & 5 \\
\hline Strep pneumoniae & 2 & 0 \\
\hline Seratia marcesnces & 1 & 2 \\
\hline Enterobacter cloacae & 1 & 1 \\
\hline Strep pyogenes & 0 & 2 \\
\hline Staphylococcus spp & 0 & 2 \\
\hline & \multicolumn{2}{|c|}{ Plaque specimen 2 } \\
\hline Mutans streptococci group & 12 & 7 \\
\hline Strep pneumoniae & 1 & 0 \\
\hline Staphylococcus spp & 2 & 0 \\
\hline Pseudomonas spp & 1 & \\
\hline
\end{tabular}

Our result is in agreement with what Soderling et al., (2011)[16] and Ge Y et al., (2008) [4] had previously reported, despite the difference in methods of detecting the bacterial organisms. Soderling et al., (2011)[16] identified the $S$. mutans species using DNADNA hybridization technique and noted that xylitol consumption did reduce the count of salivary S.mutans. We used conventional methods of bacterial culture of both salivary and plaque specimens and S.mutans levels were reduced significantly after xylitol gum consumption in both types of specimens when compared to sorbitol gum consumption.

Xylitol has favourable anti-cariogenic properties such as increasing saliva flow and $\mathrm{pH}$ and promotes remineralisation $[11,18]$. Despite these properties the evidence for xylitol as an anti-cariogenic agent has been conflicting and a systematic review of the literature reports that the evidence is lacking with a high risk of bias and confounder effects such as fluoride and time [14]. The Cochrane review also reports that the evidence was insufficient to determine whether xylitol-containing products can prevent caries and that future high quality randomised, controlled trials were needed to show whether xylitol had a greater anti-caries effect than sorbitol. This study explored the short-term effect of xylitol compared with sorbitol on the microbial composition of saliva and plaque and found that it reduced the levels of Mutans streptococci significantly compared to sorbitol.

\section{Conclusion}

Despite the few number of participants studied that could have constituted a limitation in this study, the study confirms the fact xylitol reduces the oral bacterial flora which supports the argument the use of xylitol gum helps reduce the prevalence of dental caries.

\section{References}

[1]. Campus G, Cagetti MG, Sale S, Petruzzi M, Solinas G, et al., (2013) Six months of high dose xylitol in high risk caries subjects-a 2 year randomised, clinical trial. Clin Oral Invest. 17(3): 785-791.

[2]. Loesche WJ (1986) Role of streptococcus mutans in human dental decay. Microbial Rev. 50(4): 353-380

[3]. Kawamura Y, Hou XG, Sultana F, Miura H, Ezaki T (1995) Determination of 16sRNA sequences of streptococcus mitis and streptococcus gordonii and phylogenetic relationships among members of the genus Streptococcus. Int J of Syst Bacteriol. 45(2): 406-408.

[4]. Ge Y, Caulfield PW, Fisch GS, Li Y (2008) Streptococcus mutans and streptococcus sanguinis colonization correlated with caries experience in children. Caries Res. 42(6): 444-448.

[5]. Chen T, Yu WH, Izard J, Baranova OV, Lakshmanan A, et al., (2010) The Human Oral Microbiome Database: a web accessible resource for investigating oral microbe taxonomic and genomic information. Database (Oxford). 2010: baq013

[6]. Burt BA (2006) The use of sorbitol and xylitol sweetened chewing gum in caries control. J Am Dent Assoc. 137(2): 190-196.

[7]. Tanzer JM, Thompson A, Wen ZT, Burne RA (2006) Streptococcus mutans: fructose transport, xylitol resistance, and virulence. J Dent Res. 85(4): 369-73.

[8]. Grenby TH, Phillips A, Mistry M (1989) Studies of the dental properties of lactitol compared with five other bulk sweeteners in vitro. Caries Res. 23(5): 315-319.

[9]. Soderling E, Isokangas PK, Tenovuo J (2000) Influence of maternal xylitol consumption on acquisition of mutans streptococci by infants. J Dent Res. 79(3): 882-887.

[10]. Makinen KK, Alanen P, Isokangas P, Yi W, Boxue Z, et al., (2008) Thirtynine month xylitol chewing programme in initially 8 year old school children: a feasibility study focusing on mutans streptococci and lactobacilli. Int Dent J. 58(1): 41-50.

[11]. Milgrom P, Ly KA, Roberts MC, Rothen M, Mueller G, et al., (2006) Mutans streptococci dose response to xylitol chewing gum. J Dent Res. 85(2): 177-181.

[12]. Soderling E, Isokangas PK, Tenovuo J, Mustakallio S, Makinen KK (1991) Long term xylitol consumption and mutans streptococci in plaque and saliva. Caries Res. 25(2): 153-157.

[13]. Makinen KK, Makinen PL, Pape HR Jr, Peldyak J, Hujoel P, et al., (1996) Conclusion and review of the Michigan Xylitol programme (1986-1995) for the prevention of dental caries. Int Dent J 1996. 46(1): 22-34.

[14]. Mickenautsch S, Leal SC, Yengopal V, Bezerra AC, Cruvinel V (2007) Sugar-free chewing gum and dental caries: a systematic review. J Appl Oral Sci. 15(2): 83-88.

[15]. Carroll KC, Weinstein MP (2007) Manual and automated systems for detection and identification of microorganisms. Manual of Clinical Microbiology. (9th edn), American Society.

[16]. Söderling E, Hirvonen A, Karjalainen S, Fontana M, Catt D, et al., (2011) The effect of xylitol on the composition of the oral flora: a pilot study. Eur J Dent. 5(1): 24-31.

[17]. Saravia ME, Nelson-Filho P, Ito IY, da Silva LA, da Silva RA, et al., (2011) Morphological differentiation between S. mutansand S. sobrinuson modified SB-20culture medium. Microbiol Res. 166(1): 63-67.

[18]. Ly KA, Milgrom P, Rothen M (2008) The potential of dental-protective chewing gum in oral health interventions. J Am Dent Assoc. 139(5): 553-63.

[19]. Bahador A, Lesan S, Kashi N (2012) Effect of xylitol on cariogenic and beneficial oral streptococci: a randomized, double-blind crossover trial. Iran J Microbiol. 4 (2): 75-81. 